\title{
Tecnura
}

\section{Detección de descargas parciales a través de radiación ultravioleta en redes de media y alta tensión en Colombia}

\section{Partial Discharges Detection Through Ultraviolet Radiation in Medium and High Voltage Networks in Colombia}

\author{
Edwin Fernando Sepúlveda Durán (iD) 1, Fernando Martínez Santa (iD)2 \\ Fecha de Recepción: 27 de Agosto de 2020 \\ Fecha de Aceptación: 24 de Febrero de 2021
}

Cómo citar: Sepúlveda - Durán ., E.F. y López - Sánche Martínez - Santa ., F. (2021). Detección de descargas parciales a través de radiación ultravioleta en redes de media y alta tensión en Colombia. Tecnura, 25(68), 63-78. https:/ / doi.org/10.14483/22487638.17991

\section{Resumen}

Objetivo: Mostrar el alcance del uso de la técnica de medición de descargas parciales mediante radiación ultravioleta, aplicado en la infraestructura eléctrica colombiana de acuerdo con referentes internacionales; así como también definir las características de los fenómenos físicos involucrados.

Metodología: Partiendo de la bibliografía disponible, se definen las características de las descargas parciales, suministrando información de los equipos de medida y las técnicas de medición, para finalmente determinar el grado de incursión de esta técnica en la industria colombiana.

Resultados: Colombia es un consumidor de tecnología desarrollada por otros países (Gallego y Gutiérrez, 2016), específicamente las inspecciones coronográficas son realizadas por grandes industrias del sector energético; este tipo de medición es poco frecuente en la industria nacional, habiendo muy pocas empresas que ofrecen el servicio.

Conclusiones: La técnica de coronografía es muy costosa para ser usada en pequeñas empresas, sin embargo, en grandes industrias se justifica su aplicación al prevenir grandes paradas en procesos industriales.

Palabras clave: descarga parcial, efecto corona, espectro electromagnético, radiación ultravioleta, coronografía.

\begin{abstract}
Objective: To show the scope of using the partial discharge measurement technique through ultraviolet radiation, applied on the Colombian electrical infrastructure according to international references, as well as define the characteristics of the physical phenomena involved.

Methodology: Starting from the available bibliography, the characteristics of partial discharges are defined, providing information on measuring equipment and measurement techniques, and finally to establish the degree of incursion of this technique in the Colombian industry.

\footnotetext{
${ }^{1}$ Ingeniero eléctrico, ingeniero residente Disico S. A., Bogotá, Colombia. Contacto: residentemto@disico.com.co

${ }^{2}$ Ingeniero en Control Electrónico e Instrumentación, magíster en Ingeniería Electrónica y de Computadores. Docente asistente Universidad Distrital Francisco José de Caldas, Bogotá, Colombia. Contacto: fmartinezs@udistrital.edu.co
} 
Results: Colombia is a consumer of technology developed by other countries (Gallego y Gutiérrez, 2016), specifically the coronographic inspections are carried out by large industries in the energy sector, this kind of measurement is rare in the national industry, where there are very few companies that offer the service.

Conclusions: The coronography technique is expensive to be used in small enterprises, however, in large industries, its application is justified due to it prevents large stops in industrial processes.

Keywords: partial discharge, corona effect, electromagnetic spectrum, ultraviolet radiation, coronography.

\section{Tabla de Contenidos}

Página

\section{INTRODUCCIÓN}

Descripción del fenómeno de descargas parciales

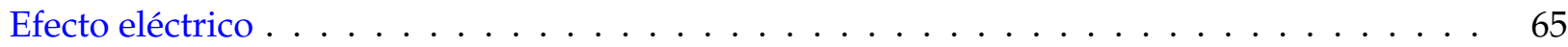

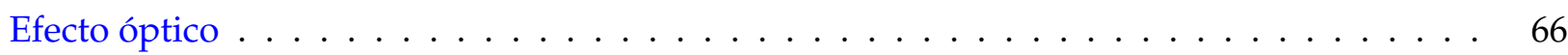

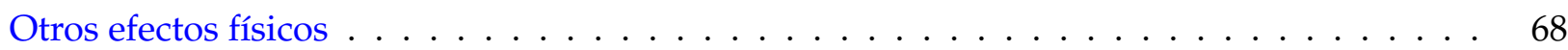

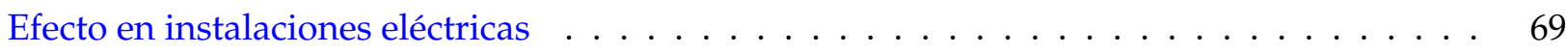

Métodos de medición y equipos . . . . . . . . . . . . . . . . . . . . 69

Desarrollo en Colombia $\ldots \ldots \ldots \ldots \ldots$. . . . . . . . . . . . . . . 71

$\begin{array}{ll}\text { CONCLUSIONES } & 72\end{array}$

$\begin{array}{lc}\text { REFERENCIAS } & 73\end{array}$

\section{INTRODUCCIÓN}

Las descargas parciales son fenómenos presentados entre dos puntos a diferente potencial eléctrico (Campuzano-Martínez, 2016,Gómez-Ramírez, 2018,Sánchez-Torres y Rocha-Millan, 2016); sucede cuando el campo electromagnético hace que se rompa la rigidez dieléctrica del medio aislante (Christina et al., 2017, Ooi, Yashima y Okamoto, 2017,Wu, Jin, Mor y Smit, 2017,Forero, Rojas y Cortés, 2015 ). Están presentes en la infraestructura eléctrica, principalmente en aisladores, conductores, estructuras de soporte y bujes de transformadores. Producto de estas descargas resultan: compuestos residuales (Janda, Martišovitš, Hensel y Machala, 2016; Zeng et al., 2015) por efecto de ionización del medio circundante; emisión de fotones en el rango ultravioleta del espectro electromagnético, y descomposición de materiales aislantes.

Las técnicas de detección han venido evolucionando y algunos recursos tecnológicos mejoran la precisión de la detección y categorización de las fallas en elementos eléctricos, logrando de manera 
efectiva anticiparse a estas (Lughofer y Sayed-Mouchaweh, 2019, Wang, 2016).

Un nuevo método de detección de fallas en cuanto a descargas parciales es la coronografía (Monrolin, Praud y Plouraboué, 2018). En este artículo se hizo una revisión de dicha técnica, así como también del uso que se le ha dado en la industria eléctrica colombiana.

\section{Descripción del fenómeno de descargas parciales}

Durante la aparición del fenómeno de descargas parciales están involucrados varios aspectos físicos, que se describirán durante el desarrollo de este artículo.

\section{Efecto eléctrico}

El efecto corona es la acumulación de campo eléctrico en las puntas de los conductores (Newport, 2018); es proporcional al nivel de tensión (Riebel, Radtkey Loos, 2002), y causa la ionización del aire circundante a este material (Florkowska et al., 2012, Nagi y Kunicki, 2017).

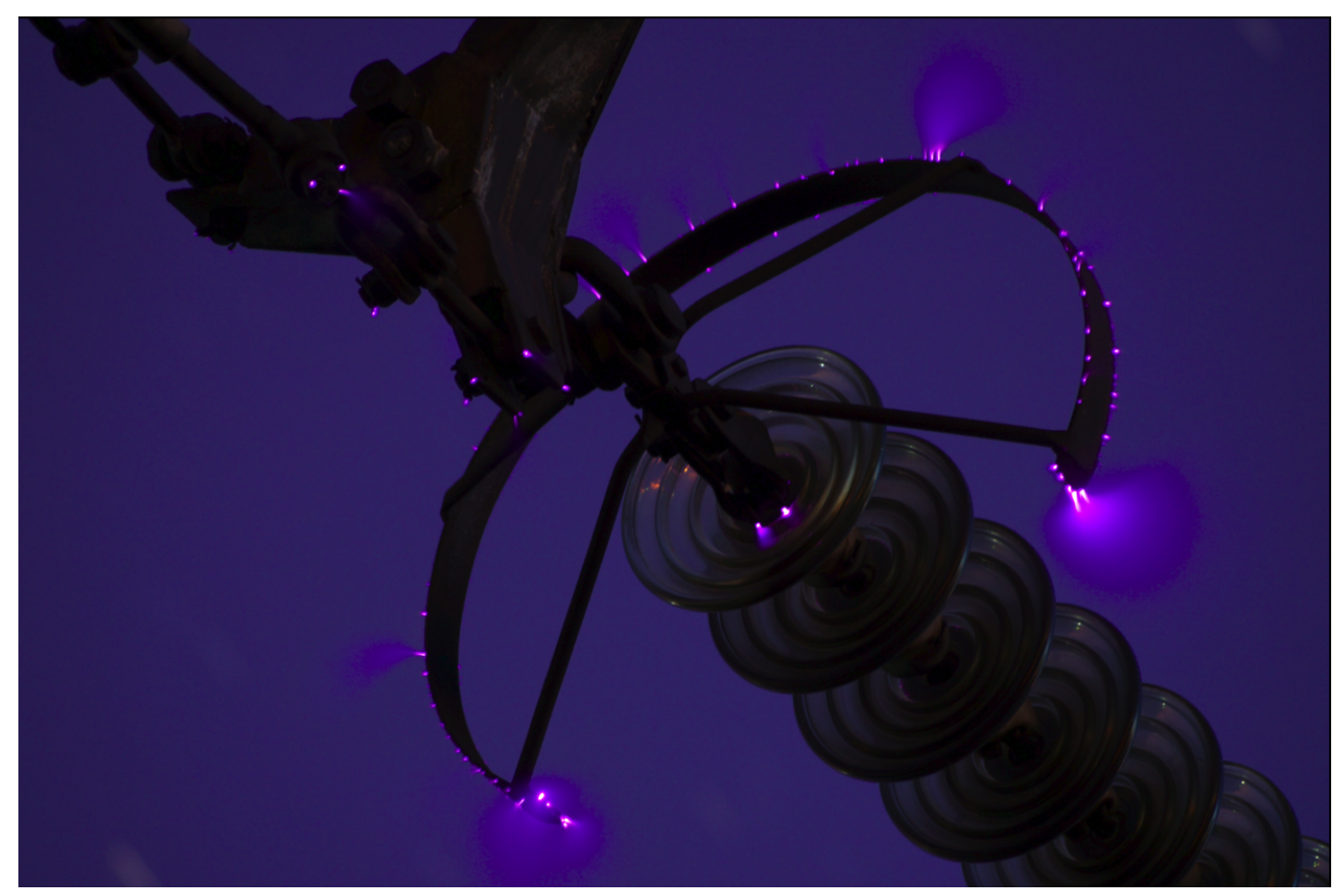

Figura 1. Anillo anticorona en una línea de $500 \mathrm{kV}$

Fuente: Andrés Granero (2016).

El arco eléctrico ocurre cuando el material aislante (cerámica, plástico o aire) se fractura (Liebowitz, 2018, Castaño, Gómez y Durango, 2016) a causa principalmente del aumento del campo eléc- 
trico (Raizer, 2017) a niveles críticos, generando conducción de corriente a través del medio y dando origen a descargas parciales entre dos puntos a diferente potencial.

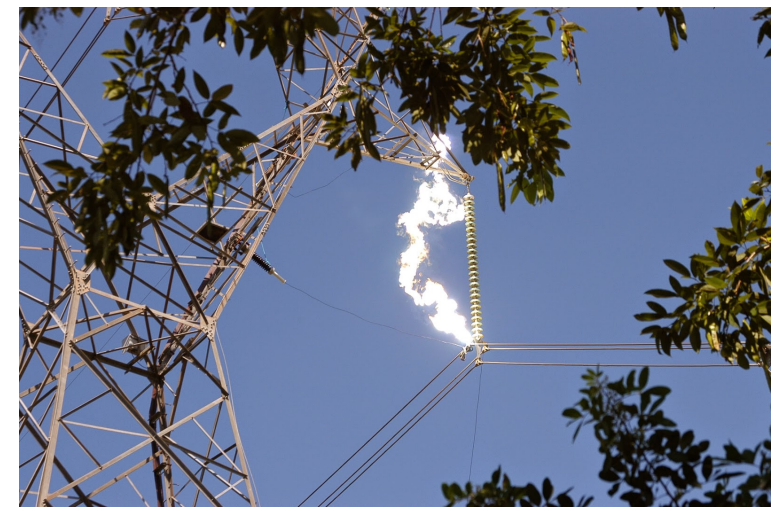

Figura 2. Arco eléctrico en cadena de aisladores

Fuente: Guido Copa (2013).

Inicialmente en los materiales aislantes se presentan imperfecciones, poros o fisuras. Para el caso del aire se presenta una ionización:

$$
V d=m d \cdot \delta \cdot \in r a \cdot \ln D r
$$

En donde $V d$ corresponde a la tensión critica disruptiva, $m d$ es una constante asignada para el conductor objeto de estudio, $\in$ ra es la rigidez dieléctrica del aire, por su parte $r$ es el radio del conductor en cm y $D$ es la distancia equivalente entre conductores en cm (Sánchez-Torres y RochaMillan, 2016).

\section{Efecto óptico}

Mientras la detección de descargas parciales por medición de ultrasonido tiene un concepto conocido y ampliamente desarrollado (Zhong, Yuan, Yao y Pan, 2019), la inspección visual de estos fenómenos está en pleno desarrollo (Ren, Song, Zhuang y Yang, 2018). Una de las ventajas más importantes de la inspección coronográfica de descargas parciales frente a una medición por ultrasonido es la precisión para identificar el elemento, o parte de este, que se encuentra con problemas. Las descargas parciales en su etapa inicial son casi imperceptibles en el día y apenas evidenciables en la noche (Newport, 2018). De manera similar a como la termografía aprovecha la radiación infrarroja (Vollmer y Möllmann, 2017) para detectar anomalías en el funcionamiento de equipos, la coronografía usa la energía en el rango ultravioleta (Frącz, Zmarzły y Boczar, 2015) del espectro electromagnético.

Las descargas de corona se generan en el espectro en el espacio de los $200 \mathrm{~nm}$ a los $400 \mathrm{~nm}$, fuera completamente del espectro visible para el ojo humano (Kozioł, Wotzka, Boczar y Frącz, 2016, 


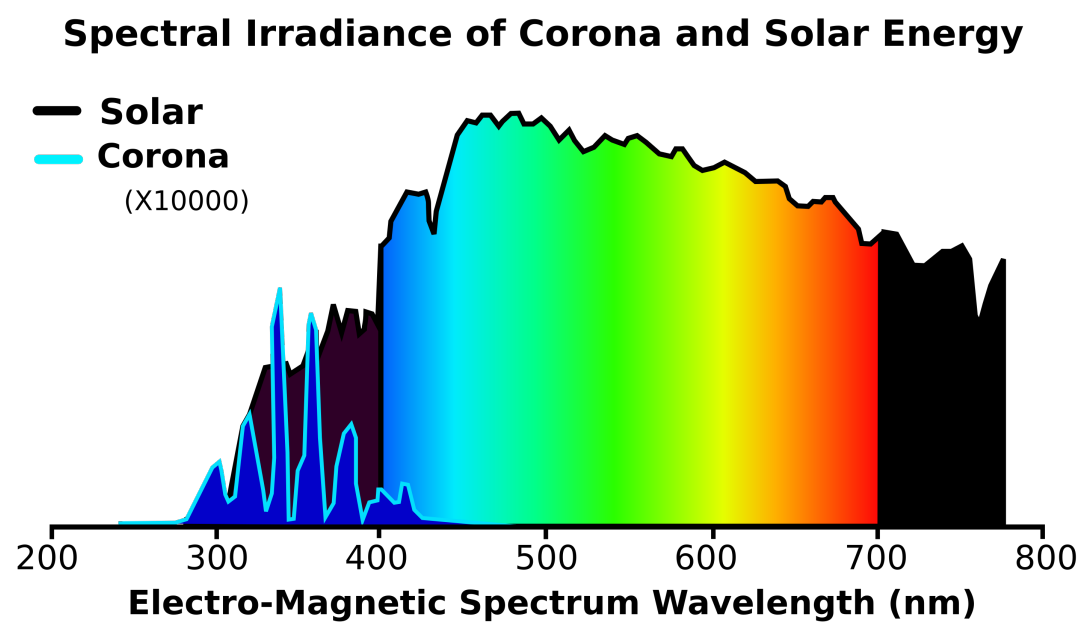

Figura 3. Ubicación de efecto corona en el espectro electromagnético

Fuente: Wallis (2015).

Newport, 2018). El sol es una fuente permanente de luz ultravioleta, lo que dificulta las inspecciones en días muy soleados; sin embargo, se han desarrollado filtros en cámaras que permiten captar el espectro en el umbral generado por las descargas parciales (Uvirco, 2015, Zhang y Wu, 2015). En estas cámaras la luz atraviesa una ventana de cuarzo; el cristal normal no permite el paso de luz ultravioleta (Chen et al., 2015) luego la luz es separada y procesada de manera independiente; la luz visible recibe un tratamiento como una imagen decolor normal para que la luz UV sea superpuesta en la imagen como pixeles de luz monocromática, visibles para que se pueda detectar el origen de la falla (Sonel, 2019, Ulirvision, 2019).

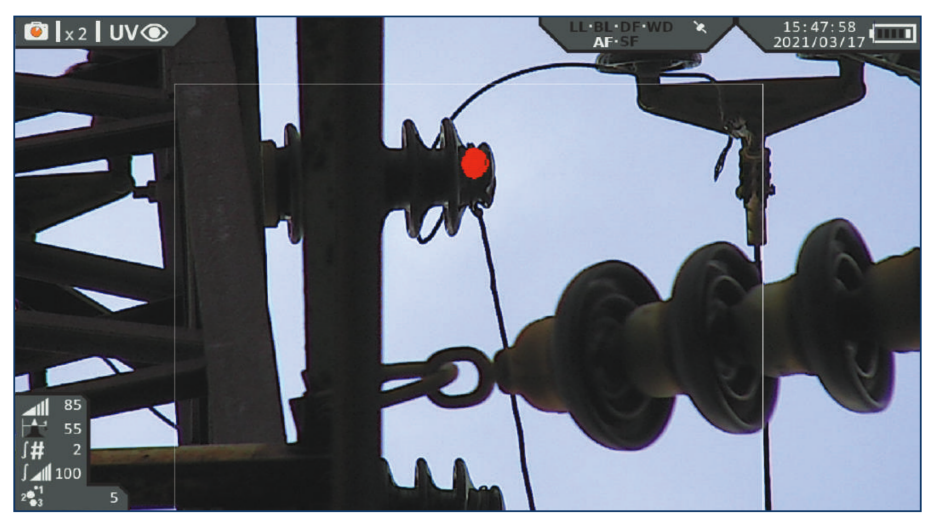

Figura 4. Imagen resultante en cámaras de coronografía

Fuente: Uvirco Technologies (2015). 


\section{Otros efectos físicos}

Para que suceda el proceso de descarga parcial se ven involucrados varios factores en la infraestructura eléctrica entre los cuales está la diferencia de potencial o nivel de tensión, el tipo de material aislante, las condiciones ambientales en las que se encuentre, entre otras (Raymond, Illias y Mokhlis, 2015). Por efecto del campo eléctrico resultante, los átomos circundantes a la zona energizada tienden a ionizarse, liberando o recibiendo electrones; estos iones pasan a atraerse o liberarse (Charry, 2018) por acción del campo electromagnético; producto de las interacciones entre iones son liberados fotones los cuales hacen visible el efecto corona en la noche, este efecto visual viene acompañado de un ruido silbante y un olor a ozono producto de la interacción de los átomos de oxigeno presentes en la atmosfera (Donini et al., 2016).

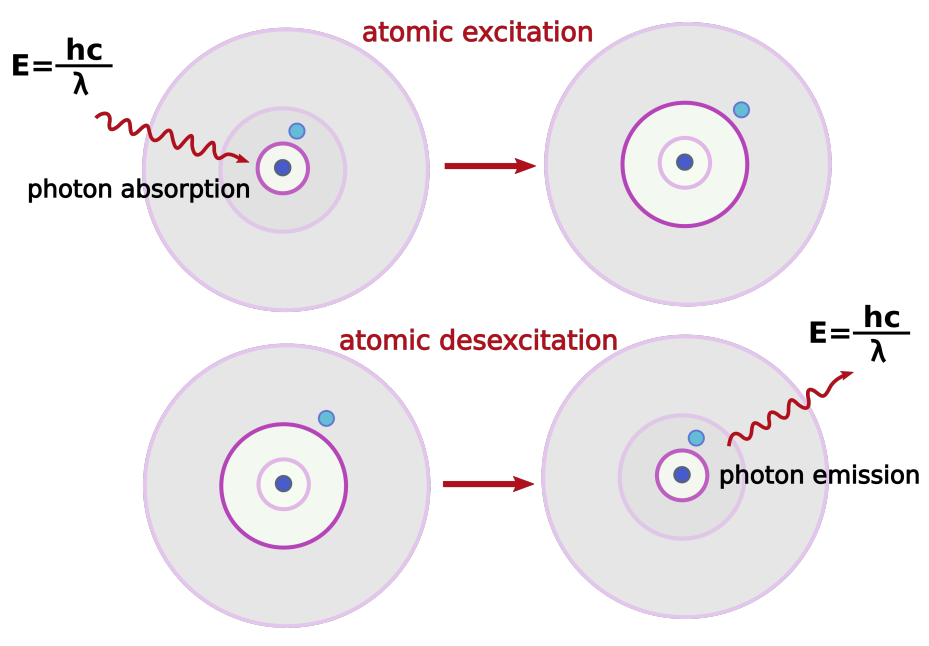

Figura 5. Emisión y absorción de fotones

Fuente: Astronoo (2013).

Como resultado de las interacciones entre los iones del aire circundante y de los materiales de la estructura eléctrica energizada, se generan materiales corrosivos (Charry, 2018), entre ellos el gas ozono, que es formado y puede ser detectado por su olor característico (este es tóxico). Para su generación, durante la emisión de fotones algunos tienen los requisitos energéticos necesarios para romper el doble enlace de los átomos de oxígeno (Lukes et al., 2005).

$$
O_{2}+h v=0+0
$$

Donde $h v$ es la energía del fotón. Otro material resultante es el óxido de nitrógeno, compuesto tóxico e irritante, producido por las interacciones entre átomos de oxígeno y nitrógeno. La generación de estos compuestos y otros ácidos depende de los materiales de los componentes aislantes. 


\section{Efecto en instalaciones eléctricas}

Los efectos en las instalaciones eléctricas pueden ser muy perjudiciales, entre ellos se encuentran: pérdidas de potencial eléctrico durante la liberación de energía en una descarga parcial (Liao, 2016), deterioro de conductores y componentes metálicos por erosión (Shahsavarian y Shahrtash, 2015) y en el caso de aisladores craking (fisuras en aisladores) y tracking (creación de caminos conductores en el aislador) (Espitia, 2017).

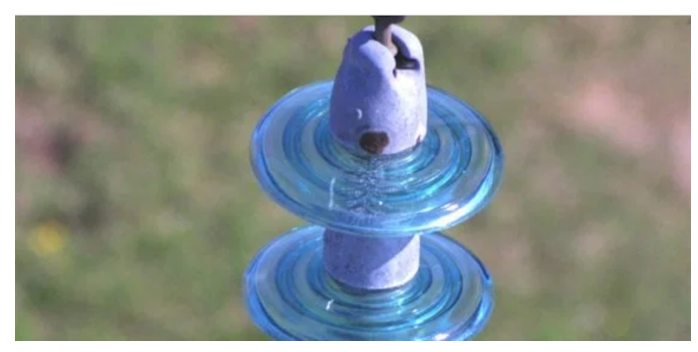

Figura 6. Degradación de aislador por efecto de descargas parciales

Fuente: Espitia (2017).

Respecto al funcionamiento de las redes se puede presentar interrupción del fluido eléctrico por falla en componentes seccionadores y radiointerferencia en un amplio espectro desde los 0,5 $\mathrm{MHz}$ hasta los $30 \mathrm{MHz}$ (Au, Agba y Gagnon, 2015).

\section{Métodos de medición y equipos}

El estudio de descargas parciales ha tenido un gran avance con equipos de medición por ultrasonido; los patrones de amplitud y frecuencia permiten al inspector caracterizar el tipo de anomalía presentada en la red eléctrica; sin embargo, lograr determinar con precisión el elemento en falla es complejo (Xie et al., 2016). La técnica de inspección por ultrasonido cuenta con niveles de certificación para personal avalados por la American Society for Nondestructive Testing (ASNT); adicionalmente, varias instituciones en Colombia dan capacitaciones y certificaciones (Dayal, Benedict, Bhatnagar y Harper, 2018).

La inspección a través de equipos de adquisición de imágenes aún está en desarrollo, con buenos avances en detección de descargas parciales en escenarios de luz de día y con una amplia gama de herramientas que facilitan al inspector las condiciones necesarias para una mejor localización y caracterización de la falla (Ofil, 2019, Uvirco, 2019). Entre las características de las cámaras desarrolladas actualmente se encuentran (Sonel, 2019, Uvirco, 2019): equipos más livianos y portátiles, resultados en tiempo real, operación diurna, software de análisis de información, sensibilidad aun a grandes distancias (desde $15 \mathrm{~m}$ ), detección de zonas de descarga cada vez más pequeñas. Un aspecto importante en desarrollo es la certificación de los procedimientos, protocolos y niveles de inspección, papel 
que han liderado las empresas desarrolladoras de esta tecnología, las cuales a través de capacitaciones y conferencias han mostrado a la industria las ventajas de cámaras de inspección de descargas parciales (Newport, 2018, Wallis, 2015).

Sin embargo, las cámaras coronográficas aún deben enfrentar las condiciones que dificultan una buena adquisición de imágenes; entres estos factores están: condiciones superficiales del material (Ai et al., 2015), polen, insectos, gotas de agua o cristales de nieve, humedad, velocidad del viento, temperatura ambiente, densidad del aire, presión atmosférica y distancia de detección.

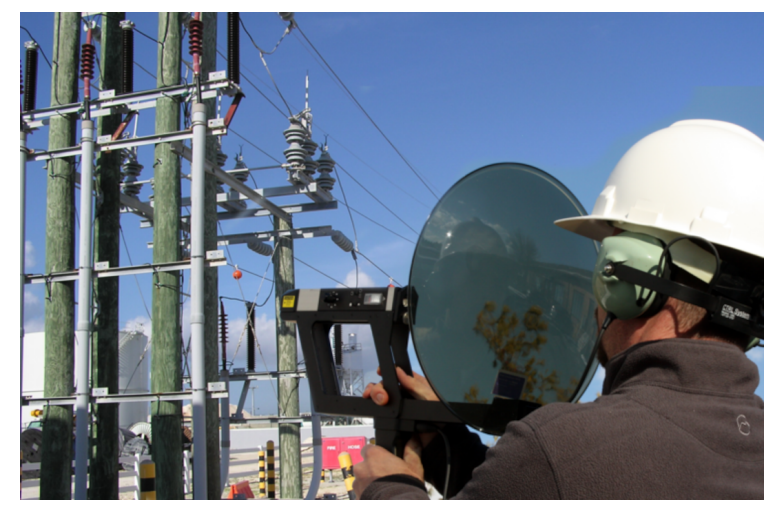

Figura 7. Detección de descargas parciales por ultrasonido

Fuente: Sedisa (2018).

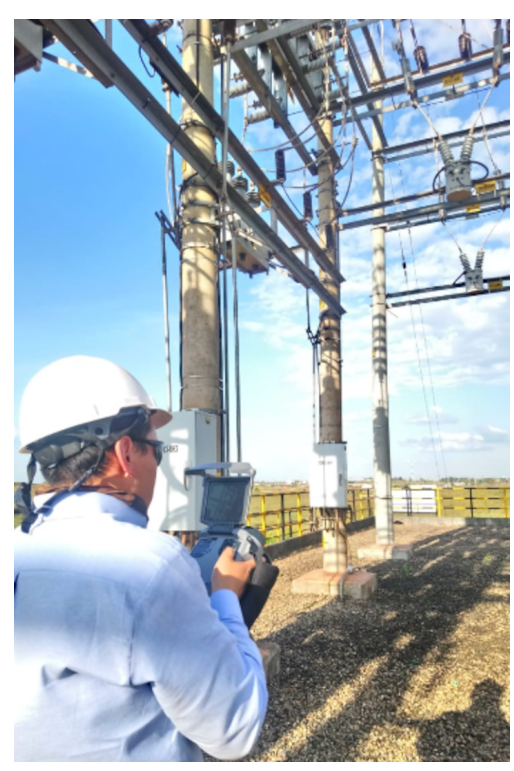

Figura 8. Detección de descargas parciales con cámaras sensibles a radiación UV

Fuente: elaboración propia. 


\section{Desarrollo en Colombia}

Como muestra del desarrollo del mantenimiento predictivo en la industria colombiana se encuentra la aplicación de la termografía, análisis de vibraciones y calidad de energía, por medio de las cuales se puede predecir fallas y anticiparse al correctivo (Pilco, Pechortinta y Cañarí, 2018). Así es como la detección de descargas parciales con cámaras sensibles a radiaciones UV entra a desempeñar un papel importante en la confiabilidad de equipos y redes en la industria, iniciando su aplicación en grandes empresas del sector de hidrocarburos y distribución de energía (Sánchez-Torres y RochaMillan, 2016). Precisamente, estos sectores de la industria están en capacidad de costear un servicio de coronografía, alquilar o comprar un equipo y capacitar personal para adelantar una inspección. La tabla I muestra los costos de aplicación de la técnica de acuerdo con tarifas de dos empresas colombianas (Erasmus, 2019, Transequipos, 2019).

Tabla I. Costos de la coronografía en inspecciones

\begin{tabular}{cccccc}
\hline Empresa & Cámara & $\begin{array}{c}\text { Precio } \\
\text { equipo }\end{array}$ & $\begin{array}{c}\text { Precio } \\
\text { alquiler/día }\end{array}$ & $\begin{array}{c}\text { Precio } \\
\text { servicio/día }\end{array}$ & Capacitación \\
\hline \multirow{2}{*}{ Erasmus } & Corocam & $€ 50.600,00$ & $€ 320,00$ & $€ 550,00$ & $€ 7.800,00$ \\
& 6D Uvirco & & & & \\
\hline \multirow{2}{*}{ Transequipos } & Uvollé VC & $€ 37.800,00$ & No disponible & $€ 455,00$ & No disponible \\
& Ofil & & & & \\
\hline
\end{tabular}

Fuente: elaboración propia.

Otro gran papel cumplen los principales distribuidores de equipos de medición en alianza con los certificadores e instructores de técnicas de mantenimiento predictivo, están a cargo de actualizar los protocolos de medición, caracterizar los tipos de falla y validar su criticidad en componentes de instalaciones eléctricas de alta y media tensión (Wallis, 2015, Pérez, Rojas, León y Cantor, 2015). Dentro de los elementos objeto de inspección están (Uvirco, 2013) las líneas de transmisión (aisladores y conductores), las estructuras de soporte, los transformadores de corriente y voltaje, los bujes de transformadores.

Por último, es importante reiterar el hecho de que el avance tanto nacional como internacional de la coronografía aún esta en desarrollo; no se puede contar, como en el caso de los demás métodos de inspección predictivos, con escalafones o niveles de certificación y protocolos avalados porinstituciones como la ASNT. Sin embargo, como ya se ha mencionado, la coronografía tendrá un alto impacto en la predicción de fallas en componentes eléctricos de alta y media tensión. 


\section{CONCLUSIONES}

La medición de descargas parciales mediante el uso de cámaras de detección de emisión ultravioleta permite de manera precisa identificar el componente que está averiado, situando con pixeles de luz monocromática las manifestaciones de emisión de fotones, microdescargas y efectos corona en la imagen digital de equipo objeto de inspección. Este resultado no era posible con una inspección por ultrasonido, aunque se podía caracterizar el tipo de fenómeno presentado y determinar su magnitud; se requería de la desenergización del circuito para hacer una evaluación en sitio para una posterior corrección, en caso de que la anomalía en el material aislante fuera evidente.

En Colombia los costos de equipos e inspecciones hacen que los principales clientes de estas mediciones sean grandes industrias del sector de hidrocarburos y energía; estas empresas pueden adquirir los equipos que oscilan entre $€ 37000$ y $€ 50$ 000; su personal encargado de la administración del mantenimiento cuenta con capacitaciones avanzadas para el análisis de fallas asociadas a descargas parciales, y adicionalmente tienen una o varias personas con la capacidad de realizar la operación del equipo y detectar en sitio una posible falla en sus sistemas eléctricos.

Por su parte, las medianas empresas tienen la opción de implementar en su plan de mantenimiento la inspección de descargas parciales por coronografía, adquiriendo servicios de inspección con empresas especializadas en mantenimiento predictivo, este servicio está disponible por valores entre los $€ 450$ y $€ 550$; la empresa presta el servicio y entrega al cliente un informe completo donde recomienda los correctivos pertinentes.

Los principales impulsores de la expansión de esta tecnología son las empresas extranjeras desarrolladoras de equipos de medición; estas cuentan con laboratorios dedicados a pruebas con distintos materiales para aumentar las prestaciones de sus equipos, también son las encargadas de dar a conocer las características de nuevas tecnologías y, como son eficaces en la implementación en programas de mantenimiento, tienen conocimiento y experiencia para capacitar a las organizaciones importadoras de tecnología. Este es el caso de Colombia, donde las empresas que venden y alquilan los equipos ya están en capacidad de orientar en la primera etapa a la industria colombiana en el uso de la técnica de coronografía.

La coronografía, o detección de descargas parciales por emisión de radiación ultravioleta, entra a formar parte de las herramientas que tiene la industria colombiana para proteger sus activos. Directores de mantenimiento y personal técnico tienen en sus manos la tecnología para detectar fallas en componentes eléctricos de manera temprana, generando rutinas de inspección, y decidiendo es el momento oportuno para ejecutar una reparación. El país tiene la oportunidad de hacer propia esta técnica de inspección; la invitación es para que los desarrolladores de iniciativas con aplicaciones industriales pasen de las manos de distribuidores de tecnología a laboratorios de universidades y grupos de investigación. 


\section{REFERENCIAS}

[Ai et al., 2015] Ai, J., Jin, L., Zhang, Y., Tian, Z., Peng, C. y Duan, W. (2015). Detecting partial discharge of polluted insulators based on ultraviolet imaging. En 2015 IEEE 11th International Conference on the Properties and Applications of Dielectric Materials (ICPADM) (pp. 456-459). IEEE. https://doi.org/10.1109/ICPADM.2015.7295307个Ver página 70

[Astronoo.com, 2013] Astronoo.com (2013). Principio de absorción y de emisión atómica. Recuperado de http://www.astronoo.com/es/articulos/ principio-absorcion-emision-atomica.html $\uparrow$ Ver página

[Au, Agba y Gagnon, 2015] Au, M., Agba, B. L. y Gagnon, F. (2015). A model of electromagnetic interferences induced by corona discharges for wireless channels in substation environments. IEEE Transactions on Electromagnetic Compatibility, 57(3), 522-531. https : / / doi . org/10 . 110 9/ TEMC . $2015.2402638 \uparrow$ Ver página 69

[Campuzano-Martínez, 2016] Campuzano-Martínez, I. R. (2016). Diagnóstico de generadores eléctricos de potencia con técnicas de monitoreo en línea y fuera de línea. Información Tecnológica, 27(2), 11-20. https://doi.org/10.4067/S0718-07642016000200003 个Ver página 64

[Castaño, Gómez y Durango, 2016] Castaño, C. G., Gómez, A. A. y Durango, J. J. M. (2016). Modelos de horno de arco eléctrico para estudios del efecto flicker. Tecnura, 20(48), 15-28. $\uparrow$ Ver página 65

[Charry, 2018] Charry, J. M. (2018). Air ions: physical and biological aspects. Londres: CRC Press. https://doi.org/10.1201/9781351069571 个Ver página 68

[Chen et al., 2015] Chen, H., Liu, K., Hu, L., Al-Ghamdi, A. A. y Fang, X. (2015). New concept ultraviolet photodetectors. Materials Today, 18(9), 493-502. https: / / doi . org/10.1016/j . mattod. $2015.06 .001 \uparrow$ Ver página 67

[Christina et al., 2017] Christina, A. J., Salam, M. A., Rahman, Q. M., Arifin, M. S., Wen, F., Ang, S. P. y Hasan, S. (2017). Partial discharge modeling with increasing applied voltages across different voidsizes. En 2017 4th International Conference on Advances in Electrical Engineering (ICAEE) (pp. 22-25). IEEE. https://doi.org/10.1109/ICAEE.2017.8255320 个er página 64

[Copa, 2013] Copa, G. (2013). Cicloconvertidores. Recuperado de http://ingcopa.blogspot. $\mathrm{com} / \uparrow$ Ver página

[Dayal, Benedict, Bhatnagar y Harper, 2018] Dayal, V., Benedict, Z. G., Bhatnagar, N. y Harper, A. G. (2018). Development of composite calibration standard for quantitative NDE by ultrasound and thermography. AIP Conference Proceedings, 1949(1), 60006. Melville: AIP Publishing. https: //doi.org/10.1063/1.5031552 个Ver página 69 
[Donini et al., 2016] Donini, A., Spezie, R., Cortina, R., Piana, E. A. y Turri, R. (2016). Accurate prediction of the corona noise produced by overhead transmission lines. En 2016 AEIT International Annual Conference (AEIT), 1-6. IEEE. https://doi.org/10.23919/AEIT.2016.7892760 $\uparrow$ Ver página 68

[Erasmus, 2019] Erasmus (2019). UViRCO Technologies, portafolio. Marcas representadas. Recuperado de https://www.erasmus.com.co/index.php/es/marcas-representadas/uvirco $\uparrow$ Ver página 71

[Espitia, 2017] Espitia, C. (2017). Inspección de líneas de transmisión y distribución mediante coronografía. La Nota Energética. Transequipos.com. Recuperado de https: / / transequipos . com/ la-nota-energetica/ $\uparrow$ Ver página 69

[Florkowska et al., 2012] Florkowska, B., Roehrich, J., Zydron, P., Florkowski, M. y Rybak, A. (2012). Interaction of conductor with polymeric materials (XLPE/EPR) at partial discharges. IEEE Transactions on Dielectrics and Electrical Insulation, 19(6), 2119-2127. https : / / do i org/10 .1109/TDEI . $2012.6396972 \uparrow$ Ver página

[Forero, Rojas y Cortés, 2015 ] Forero, M. C., Rojas, H. E. y Cortés, C. A. (2015). Aplicación de la transformación local polinomial de Fourier en el análisis de descargas parciales. Tecnura, 19, 15-23. $\uparrow$ Ver página 64

[Frącz, Zmarzły y Boczar, 2015] Frącz, P., Zmarzły, D. y Boczar, T. (2015). Characteristic of surface partial discharges measured with ultraviolet camera. Acta Physica Polonica Series a, 127(3), 715-718. https://doi.org/10.12693/APhysPolA.127.715 †er página 66

[Gallego y Gutiérrez, 2016] Gallego, J. M. y Gutiérrez, L. H. (2016). El Sistema Nacional de Calidad en Colombia: un análisis cualitativo del desarrollo del sistema. Washington: Inter-American Development Bank. https://doi.org/10.18235/0000419 ^Ver página 63,64

[Granero, 2016] Granero, A. (2016). Efecto corona en líneas de alta tensión. Recuperado de http://imseingenieria.blogspot.com/2016/08/ efecto-corona-en-lineas-de-alta-tension.html $\uparrow$ Ver página

[Gómez-Ramírez, 2018] Gómez-Ramírez, G. A. (2018). Measurement of partial discharge in power transformers under international standards IEC and IEEE. Revista Tecnología en Marcha, 31(1), 7080. https://doi.org/10.18845/tm.v31i1.3498个Ver página 64

[Janda et al., 2016] Janda, M., Martišovitš, V., Hensel, K. y Machala, Z. (2016). Generation of antimicrobial NO X by atmospheric air transient spark discharge. Plasma Chemistry and Plasma Processing, 36(3),767-781. https: / / doi.org/10.1007/s11090-016-9694-5个Ver página 
[Kozioł, Wotzka, Boczar y Frącz, 2016] Kozioł, M., Wotzka, D., Boczar, T. y Frącz, P. (2016). Application of optical spectrophotometry for analysis of radiation spectrum emitted by electric arc in the air. Journal of Spectroscopy, 2016. https :// doi .org/10.1155/2016/1814754 个Ver página 66

[Liao, 2016] Liao, Y., Feng, B., Gu, X., Sun, T., Xu, Y. y Zhang, Z. (2016). Application of the online partial discharge monitoring for the EHV XLPE cable system. En 2016 International Conference on Condition Monitoring and Diagnosis (CMD) (pp. 896-899). IEEE. https://doi.org/10.1109/ CMD . 2016.7757966 ^Ver página 69

[Liebowitz, 2018] Liebowitz, H. (2018). Fracture of metals: an advanced treatise. Ámsterdam: Elsevier. $\uparrow$ Ver página 65

[Lughofer y Sayed-Mouchaweh, 2019] Lughofer, E. y Sayed-Mouchaweh, M. (2019). Predictive maintenance in dynamic systems: advanced methods, decision support tools and real-world applications. Suiza: Springer. https://doi.org/10.1007/978-3-030-05645-2个Ver página 65

[Lukes et al., 2005] Lukes, P., Clupek, M., Babicky, V., Janda, V. y Sunka, P. (2005). Generation of ozone by pulsed corona discharge over water surface in hybrid gas-liquid electrical discharge reactor. Journal of Physics D: Applied Physics, 38(3), 409. https: //doi .org/10.1088/0022-3727/38/ 3/010 个Ver página 68

[Monrolin, Praud y Plouraboué, 2018] Monrolin, N., Praud, O. y Plouraboué, F. (2018). Revisiting the positive DC corona discharge theory: Beyond Peek's and Townsend's law. Physics of Plasmas, 25(6), 63503. https://doi.org/10.1063/1.5031780 个er página 65

[Nagi y Kunicki, 2017] Nagi, Ł. y Kunicki, M. (2017). Ionizing radiation generated by the electrical discharges from medium and high voltage in the air. En 2017 IEEE International Conference on Environment and Electrical Engineering and 2017 IEEE Industrial and Commercial Power Systems Europe (EEEIC/IECPS Europe) (pp.1-5). IEEE. https : / / doi .org/10.1109/EEEIC.2017. 7977449 $\uparrow$ Ver página 65

[Newport, 2018] Newport, R. (2018). Coronography and termography. En Jornada de capacitación en equipos para detección de fallas - Erasmus, 17 de agosto de 2018. Bogotá, Colombia. $\uparrow$ Ver página $65,66,70$

[Ofil, 2019] Ofil (2019). Handheld high-definition Corona Camera DayCor® Uvollé. Ofil Systems Products - Handheld. Recuperado de https://ofilsystems.com/pro/ daycor-uvolle-compact-daytime-corona-camera/ $\uparrow$ Ver página 69

[Ooi, Yashima y Okamoto, 2017] Ooi, K., Yashima, M. y Okamoto, T. (2017). Investigation of partial discharge life characteristics of twisted pair of enamelled wires. En 2017 International Symposium on Electrical Insulating Materials (ISEIM), 1, 243-246. IEEE. https : / / doi .org/10.23919/ ISEIM. $2017.8088732 \uparrow$ Ver página 64 
[Pérez, Rojas, León y Cantor, 2015] Pérez, C. D., Rojas, H. E., León, A. F. y Cantor, L. F. (2015). Crecimiento de algas sobre aisladores de media tensión y su impacto sobre el desempeño eléctrico. Tecnura, 19, 24-32. $\uparrow$ Ver página 71

[Pilco, Pechortinta y Cañarí, 2018] Pilco, E., Pechortinta, Y. y Cañarí, J. (2018). Características de la gestión del mantenimiento industrial: revisión de la literatura. [Trabajo de grado]. Universidad Privada del Norte. Trujillo, Perú. $\uparrow$ Ver página 71

[Raizer, 2017] Raizer, Y. (2017). Spark discharge. Londres: Routledge. $\uparrow$ Ver página 66

[Raymond, Illias y Mokhlis, 2015] Raymond, W. J. K., Illias, H. A. y Mokhlis, H. (2015). Partial discharge classifications: Review of recent progress. Measurement, 68, 164-181. https: / / doi . org/ $10.1016 / j$.measurement.2015.02.032 个Ver página 68

[Ren, Song, Zhuang y Yang, 2018] Ren, M., Song, B., Zhuang, T. y Yang, S. (2018). Optical partial discharge diagnostic in SF6 gas insulated system via multi-spectral detection. ISA Transactions, 75, 247-257. https://doi.org/10.1016/j.isatra.2018.02.008 个Ver página 66

[Riebel, Radtkey Loos, 2002] Riebel, U., Radtke, R. y Loos, R. (2002). An experimental investigation on corona quenching. Journal of Electrostatics, 54(2), 159-165. https: / / doi . org/10.1016/ S0304-3886(01) 00175-9 个Ver página 65

[Sánchez-Torres y Rocha-Millan, 2016] Sánchez-Torres, A. y Rocha-Millan, G. M. (2016). Diseño de un protocolo de medición de efecto corona en sistemas eléctricos de media y alta tensión utilizando cámara coronográfica parala empresa Lyansa Electrica LTDA. [Trabajo de grado]. Universidad de los Llanos. Villavicencio, Colombia. $\uparrow$ Ver página $64,66,71$

[Sedisa, 2018] Sedisa (2018). Inspecciones eléctricas con ultrasonido acústico. Recuperado de http://www.sedisa.com.pe/servicios/sin-categoria/ inspecciones-electricas-con-ultrasonido-acustico $\uparrow$ Ver página

[Shahsavarian y Shahrtash, 2015] Shahsavarian, T. y Shahrtash, S. M. (2015). Modelling of aged cavities for partial discharge in power cable insulation. IET Science, Measurement $\mathcal{E}$ Technology, 9(6), 661-670. https://doi.org/10.1049/iet-smt.2014.0222 个Ver página 69

[Sonel, 2019] Sonel (2019). Cámara de descargas en Corona UV-260. Sonel Dispositivos Medidas de descargas parciales. Recuperado de https://www.sonel.pl/es/producto/ corona-camera-uv260_7Bp1/ 个Ver página 67,69

[Transequipos, 2019] Transequipos (2019). Inspección coronográfica ultravioleta. Confiabilidad en Equipos Eléctricos. Transequipos.com. Recuperado de https://transequipos.com/ inspeccion-coronografica/ $\uparrow$ Ver página 71 
[Ulirvision, 2019] Ulirvision (2019). TD100 Corona Camera. Recuperado de http://www . ulirvision.co.uk/corona-camera-uvsee-td100.html $\uparrow$ Ver página 67

[Uvirco, 2019] Uvirco Technologies (2019). CoroCAM® 6D Economical Daylight Corona Imaging Camera Brochure. Recuperado de https://www.uvirco.com/wp-content/uploads/2019/06/ CoroCAM-6D-Brochure-2019.2_compressed.pdf $\uparrow$ Ver página 69

[Uvirco, 2013] Uvirco Technologies (2013). CoroCAM advanced site inspection guide. Recuperado de https://slideplayer.com/slide/14580534/ Ver página 71

[Uvirco, 2015] Uvirco Technologies (2015). CoroCAM® Non Solar Blind Cameras. Recuperado de https://www. uvirco.com/products/corocam-non-solar-blind-cameras/ $\uparrow$ Ver página 67

[Vollmer y Möllmann, 2017] Vollmer, M. y Möllmann, K. P. (2017). Infrared thermal imaging: fundamentals, research and applications. Hoboken: John Wiley \& Sons. https://doi.org/10.1049/ iet-smt.2014.0222 个Ver página 66

[Wallis, 2015] Wallis, J. (2015). Making the invisible visible: UViRCO, an innovation success story: industry. CSIR Science Scope, 8(2), 80-81. $\uparrow$ Ver página 70, 71

[Wang, 2016] Wang, K. (2016). Intelligent predictive maintenance (IPdM) system-Industry 4.0 scenario. WIT Transactions on Engineering Sciences, 113, 259-268. $\uparrow$ Ver página 65

[Wu, Jin, Mor y Smit, 2017] Wu, J., Jin, H., Mor, A. R. y Smit, J. (2017). The effect of frequency on the dielectric breakdown of insulation materials in HV cable systems. En 2017 International Symposium on Electrical Insulating Materials (ISEIM), 1, 251-254. IEEE. https: / / doi . org/10.23919/ ISEIM.2017.8088734 个Ver página 64

[Xie et al., 2016] Xie, X., Mu, J., Liu, R., Meng, Q., Wu, S., Lv, P., ... , Zhu, B. (2016). Research on partial discharge detection methods for electrical equipment diagnosis. En 2016 4th International Conferenceon Machinery, Materials and Computing Technology. Dordrecht: Atlantis Press. https: //doi.org/10.2991/icmmct-16.2016.278 个Ver página 69

[Zeng et al., 2015] Zeng, F., Tang, J., Xie, Y., Zhou, Q. y Zhang, C. (2015). Experimence study of trace water and oxygen impact on SF 6 decomposition characteristics under partial discharge. Journal of Electrical Engineering \& Technology, 10(4), 1786-1795. ttps: / / doi .org/10 . 5370 / JEET. 2015. $10.4 .1786 \uparrow$ Ver página

[Zhang y Wu, 2015] Zhang, Y. y Wu, T. (2015). Optical system design of solar blind ultraviolet imaging detector. En 2015 4th National Conference on Electrical, Electronics and Computer Engineering. Dordrecht: Atlantis Press. https://doi.org/10.2991/nceece-15.2016.218 $\uparrow e r$ página 67 
[Zhong, Yuan, Yao y Pan, 2019] Zhong, D., Yuan, F., Yao, J. y Pan, J. (2019). Application analysis of ultrasonic technology in insulator contamination discharge detection. En 2019 International Conference on Computer, Network, Communication and Information Systems (CNCI 2019). Dordrecht: Atlantis Press. https://doi.org/10.2991/cnci-19.2019.20个Ver página 66 